\title{
A NEW FORMULA FOR BOX SPLINES ON THREE-DIRECTIONAL MESHES
}

\author{
EDWARD NEUMAN
}

\begin{abstract}
A new formula for $s$-variate box splines on three-directional meshes is derived. An application to evaluation of a certain multiple integral is included.
\end{abstract}

\section{INTRODUCTION}

Box splines on three-directional meshes and their translates provide a useful tool in the finite element method (see, e.g., [4-5]). This class of multivariate splines is contained in a broader class of splines introduced by de Boor and DeVore [1]. Some basic recurrence relations obeyed by the latter splines have been derived in [2]. Computations with these recursions could be cumbersome and time-consuming. In this note we offer a closed formula for multivariate box splines on three-directional meshes (see (3)).

In $\S 2$ we give notation and definitions which will be used in subsequent sections. The main result is derived in $\S 3$. An application is given in $\S 4$.

\section{Notation AND DEFINITIONS}

By $e_{j}(1 \leq j \leq s)$ we will denote the $j$ th coordinate vector in $\mathbb{R}^{s}$. Also, let $e_{s+1}=e_{1}+\cdots+e_{s}$. For $n_{1}, \ldots, n_{s+1} \in\{1,2, \ldots\}$ let $m=n_{1}+\cdots+n_{s+1}$. The three-directional mesh in $\mathbb{R}^{s}$ is the matrix

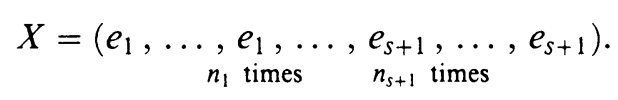

The centered box spline $B_{n}(\cdot \mid X)$ can be realized as the piecewise polynomial kernel of the distribution

$$
f \rightarrow \int_{I^{m}} f\left(\sum_{j=1}^{m} t_{j} x^{j}\right) d t, \quad f \in C_{0}^{\infty}\left(\mathbb{R}^{s}\right) .
$$

Here, $n=\left(n_{1}, \ldots, n_{s+1}\right), I=[-1 / 2,1 / 2]$, and $x^{1}, \ldots, x^{m}$ denote the columns of $X$. It is well known that $B_{n}(\cdot \mid X)$ is an $s$-variate piecewise polynomial of total degree $m-s, B_{n} \in C^{\mu-2}\left(\mathbb{R}^{s}\right)$, where $\mu$ is the minimal number of columns of $X$ to be deleted to end up with a matrix $X^{\prime}$ so that $\operatorname{rank}\left(X^{\prime}\right)<$ $\operatorname{rank}(X)$, and

Received by the editor January 25, 1993.

1991 Mathematics Subject Classification. Primary 41A15, 41A63. 


$$
\operatorname{supp} B_{n}(\cdot \mid X)=\left\{x=\sum_{j=1}^{m} t_{j} x^{j}: t_{j} \in I, x^{j} \in X\right\}
$$

The Fourier transform of $B_{n}$ is [3, (2.3)]

$$
\widehat{B}_{n}(y \mid X):=\int_{\mathbb{R}^{s}} e^{i y \cdot x} B_{n}(x \mid X) d x=\prod_{j=1}^{s+1}\left(\operatorname{Sinc} \frac{y \cdot e_{j}}{2}\right)^{n_{j}}
$$

where $y \cdot x$ is the dot product, and $\operatorname{Sinc} t=(\sin t) / t$ is the sinc function.

\section{A CLOSED FORMULA FOR $B_{n}$}

We shall now establish the following formula:

$$
B_{n}(x \mid X)=\int_{\alpha}^{\beta} \prod_{j=1}^{s+1} M_{n_{j}}\left(x_{j}+t\right) d t, \quad x_{s+1} \equiv 0 .
$$

Here, $M_{n_{j}}$ stands for the univariate central $B$-spline of degree $n_{j}-1$ with knots at $-n_{j} / 2,-n_{j} / 2+1, \ldots, n_{j} / 2$ (see, e.g., [6, Chapter 2]), $x^{T}=$ $\left(x_{1}, x_{2}, \ldots, x_{s}\right)$, and

$$
\begin{aligned}
& \alpha=\max \left\{-x_{j}-n_{j} / 2: 1 \leq j \leq s+1\right\}, \\
& \beta=\min \left\{-x_{j}+n_{j} / 2: 1 \leq j \leq s+1\right\} .
\end{aligned}
$$

For the proof of (3) we apply the inversion formula to the first and third members of $(2)$ to obtain

$$
B_{n}(x \mid X)=(2 \pi)^{-s} \int_{\mathbb{R}^{s}} e^{i x \cdot y} \prod_{j=1}^{s+1}\left(\operatorname{Sinc} \frac{y \cdot e_{j}}{2}\right)^{n_{j}} d y .
$$

Use of $[6,(1.7)]$

$$
\left(\operatorname{Sinc} \frac{y \cdot e_{s+1}}{2}\right)^{n_{s+1}}=\int_{\mathbb{R}} e^{i\left(y \cdot e_{s+1}\right) t} M_{n_{s+1}}(t) d t
$$

on (5) and the fact that $e_{s+1}=e_{1}+\cdots+e_{s}$ gives

$$
B_{n}(x \mid X)=(2 \pi)^{-s} \int_{\mathbb{R}} M_{n_{s+1}}(t)\left[\int_{\mathbb{R}^{s}} \prod_{j=1}^{s} e^{i\left(x_{j}+t\right) y_{j}}\left(\operatorname{Sinc} \frac{y_{j}}{2}\right)^{n_{j}} d y_{j}\right] d t .
$$

Combining this with

$$
\int_{\mathbb{R}} e^{i\left(x_{j}+t\right) y_{j}}\left(\operatorname{Sinc} \frac{y_{j}}{2}\right)^{n_{j}} d y_{j}=2 \pi M_{n_{j}}\left(x_{j}+t\right),
$$

we obtain

$$
B_{n}(x \mid X)=\int_{\mathbb{R}} \prod_{j=1}^{s+1} M_{n_{j}}\left(x_{j}+t\right) d t, \quad x_{s+1} \equiv 0
$$


To complete the proof, let us note that

$$
\operatorname{supp} M_{n_{j}}\left(x_{j}+\cdot\right)=\left[-x_{j}-n_{j} / 2,-x_{j}+n_{j} / 2\right],
$$

$j=1,2, \ldots, s+1$. This, in conjunction with (6), yields the assertion.

\section{An application}

We will now deal with a multiple integral which is defined by

$$
J_{s, n}:=\int_{\mathbb{R}^{s}}\left(\operatorname{Sinc} t_{1}\right)^{n_{1}} \cdots\left(\operatorname{Sinc} t_{s}\right)^{n_{s}}\left(\operatorname{Sinc}\left(t_{1}+\cdots+t_{s}\right)\right)^{n_{s+1}} d t .
$$

We show that

$$
J_{s, n}=\pi^{s} B_{n}\left(0_{s} \mid X\right)=2 \pi^{s} \int_{0}^{\beta} \prod_{j=1}^{s+1} M_{n_{j}}(t) d t,
$$

where now $\beta=\min \left\{n_{j} / 2: 1 \leq j \leq s+1\right\}$ and $0_{s}$ stands for the zero vector in $\mathbb{R}^{s}$. For the proof of (7) we make a change of variables in (5) putting $y=2 v$. It is readily seen that the Jacobian of this transformation equals $2^{s}$. Next, letting $x=0_{s}$ we obtain the first and second members of (7). To obtain the third member of (7), we use the formula (3) with $x=0_{s}$. Note that in this case the integrand of $(3)$ is an even function.

If $n=(k, \ldots, k) \in \mathbb{R}^{s+1}, k$ a positive integer, then (7) simplifies to

$$
J_{s, n}=2 \pi^{s} \int_{0}^{k / 2}\left[M_{k}(t)\right]^{s+1} d t .
$$

In particular, $J_{s, n}=\pi^{s}$ if $k=1$, and $J_{s, n}=2 \pi^{s} /(s+2)$ for $k=2$.

\section{BIBLIOGRAPHY}

1. C. de Boor and R. DeVore, Approximation by smooth multivariate splines, Trans. Amer. Math. Soc. 276 (1983), 775-788.

2. C. de Boor and K. Höllig, B-splines from parallelepipeds, J. Analyse Math. 62(1982), 99115.

3. C. K. Chui, Multivariate splines, CBMS-NSF Regional Conf. in Appl. Math., no. 54, SIAM, Philadelphia, PA, 1988.

4. P. G. Ciarlet, The finite element method for elliptic problems, North-Holland, Amsterdam, 1978.

5. F. Di Gouglielmo, Méthodes des éléments finis: une famille d'approximations des espaces de Sobolev par les translates de p-fonctions, manuscript, 1970.

6. I. J. Schoenberg, Cardinal spline interpolation, CBMS-NSF Regional Conf. in Appl. Math., no. 12, SIAM, Philadelphia, PA, 1973.

Department of Mathematics, Southern Illinois University, Carbondale, Illinois 62901-4408

E-mail address: ga38560siucrmb.bitnet 